\title{
A Novel Risk Stratification System for Predicting In-Hospital Mortality Following Coronary Artery Bypass Grafting Surgery with Impaired Left Ventricular Ejection
} Fraction

\author{
Hongyuan Lin, MD, Jianfeng Hou, MD, PhD, Hanwei Tang, MD, Kai Chen, MD, Shaoxian Guo, MD, \\ Liqing Wang, MD, Hansong Sun, MD, Zhe Zheng, MD, PhD, Shengshou Hu, MD, PhD \\ Cardiac Surgery Centre, Fuwai Hospital, Chinese Academy of Medical Sciences and Peking Union Medical College, Beijing, China
}

\section{ABSTRACT}

Background: Coronary artery disease (CAD) is the most common cause of heart failure (HF), and impaired ejection fraction $(\mathrm{EF}<50 \%)$ is a crucial precursor to HF. Coronary artery bypass grafting $(\mathrm{CABG})$ is an effective surgical solution to CAD-related HF. In light of the high risk of cardiac surgery, appropriate scores for groups of patients are of great importance. We aimed to establish a novel score to predict in-hospital mortality for impaired EF patients undergoing CABG.

Methods: Clinical information of 1,976 consecutive CABG patients with $\mathrm{EF}<50 \%$ was collected from January 2012 to December 2017. A novel system was developed using the logistic regression model to predict in-hospital mortality among patients with $\mathrm{EF}<50 \%$ who were to undergo CABG. The scoring system was named PGLANCE, which is short for seven identified risk factors, including previous cardiac surgery, gender, load of surgery, aortic surgery, NYHA stage, creatinine, and EF. AUC statistic was used to test discrimination of the model, and the calibration of this model was assessed by the Hosmer-lemeshow (HL) statistic. We also evaluated the applicability of PGLANCE to predict in-hospital mortality by comparing the $95 \%$ CI of expected mortality to the observed one. Results were compared with the European Risk System in Cardiac Operations (EuroSCORE), EuroSCORE II, and Sino System for Coronary Operative Risk Evaluation (SinoSCORE).

Results: By comparing with EuroSCORE, EuroSCORE II and SinoSCORE, PGLANCE was well calibrated (HL $P=0.311)$ and demonstrated powerful discrimination $(\mathrm{AUC}=0.846)$ in prediction of in-hospital mortality among impaired EF CABG patients. Furthermore, the $95 \%$ CI of mortality estimated by PGLANCE was closest to the observed value.

Conclusion: PGLANCE is better with predicting inhospital mortality than EuroSCORE, EuroSCORE II, and SinoSCORE for Chinese impaired EF CABG patients.

Received April 8, 2020; received in revised form May 14, 2020; accepted May 15, 2020.

Correspondence: Fianfeng Hou, MD, PbD, No. 167, North Lishi Street, Xicheng District, Beijing, China, 100037; +86-15321663719 (e-mail: lancetlin@126.com;.jjf2006111@126.com).

\section{INTRODUCTION}

Few health problems over past decades have affected the socio-medical landscape worldwide like heart failure (HF). Coronary artery disease (CAD) is the most common cause of HF [Yancy 2013]. Coronary artery bypass grafting (CABG) invariably is recommended for CAD-related $\mathrm{HF}$ disease [F WSKPA 2014]. However, for a variety of reasons, the perioperative mortality of CABG in HF patients is higher than in general patients [Velazquez 2011]. As an independent risk factor, HF was thought to be associated with a higher in-hospital mortality in clinical practice [Nashef 1999; Hu 2000; Gardner 2004; Nashef 2012]. Impaired EF (EF<50\%) is of importance to exclude HFpEF (heart failure with preserved $\mathrm{EF}, \mathrm{EF} \geq 50 \%$ ) which indicates a favorable prognosis [Ponikowski 2016]. As a crucial precursor to HF, patients with impaired EF usually have systolic or diastolic left ventricular dysfunction [Ponikowski 2016]. Hence, close attention should be paid to EF value in clinical practice. To avoid severe morbidities of HF, CAD patients with impaired EF should undergo timely revascularization therapies, including CABG. In addition, EF value is an objective and standardized parameter that is more accepted in clinical research.

Aiming to lower postoperative mortality, the risk stratification system is of critical importance to assess operative risk, which could help with risk control. Recently, many operative risk evaluation models for CABG have been established. Some are extensively utilized; examples include the model of the Society of Thoracic Surgeons (STS) [Edwards 1997], EuroSCORE [Nashef 1999], EuroSCORE II [Nashef 2012], and SinoSCORE [Zheng 2013]. But all the published models are based on general CAD patients and theoretically may not be applicable to particular patients with impaired EF. These models also mostly use data collected 10 years ago, which may not reflect the current situation [Choong 2009]. In light of lacking appropriate prediction models for impaired EF CABG patients, developing a novel risk stratification system for impaired EF patients undergoing CABG became necessary.

\section{MATERIALS AND METHODS}

Enrolled in the study were all patients $(\mathrm{N}=1978)$, whose $\mathrm{EF}$ values were impaired (LVEF $<50 \%$, which is a precursor to 
Table 1. Demographics and risk factors for univariate analysis

\begin{tabular}{|c|c|c|}
\hline Variable & Definition & $\begin{array}{l}\text { Mean } \pm \text { SD or } \\
\text { No. }(\%)\end{array}$ \\
\hline \multicolumn{3}{|l|}{ Patient-related } \\
\hline Age & - & $60.1 \pm 9.1$ \\
\hline Female & - & $293(14.8)$ \\
\hline BMI & Body mass index & $24.9 \pm 3.9$ \\
\hline History of myocardial infarction & Documented history or ECG evidence including recent myocardial infarction (<21 days) & $899(45.5)$ \\
\hline Hypertension & Documented past history or $\mathrm{SBP}>140 \mathrm{mmHg}$ and/or $\mathrm{DBP}>90 \mathrm{mmHg}$ & $1055(53.4)$ \\
\hline Diabetes mellitus & Documented past history or fulfilled the criteria of WHO 1999 & $501(25.4)$ \\
\hline Previous $\mathrm{PCl}$ & Documented history & $174(8.8)$ \\
\hline Increased serum creatinine & Measured before surgery or any previous serum creatinine $>176 \mu \mathrm{mol} / \mathrm{L}$ & $18(0.9)$ \\
\hline Previous cardiac surgery & One or more previous major cardiac operation involving opening the pericardium & $77(3.9)$ \\
\hline History of smoking & Prior history of smoking, regardless of whether the patients quit smoking & $1627(82.3)$ \\
\hline COPD & Long-term use of bronchodilators or steroids for lung disease & $9(0.5)$ \\
\hline Extracardiac arteriopathy & $\begin{array}{c}\text { Any one or more of the following: claudication, carotid occlusion or }>50 \% \text { stenosis, previous or } \\
\text { planned intervention on the abdominal aorta, and limb arteries or carotids }\end{array}$ & $146(7.4)$ \\
\hline Cerebrovascular accident & Documented past history of coma $\geq 24 \mathrm{~h}$ or central nervous system dysfunction $\geq 72 \mathrm{~h}$ & $63(3.2)$ \\
\hline Critical preoperative state & $\begin{array}{l}\text { Any one or more of the following occurring preoperatively: } \\
\text { ventricular tachycardia or fibrillation or aborted sudden death; cardiac massage; ventilation before } \\
\text { arrival in the anaesthetic room; inotropes; intra-aortic balloon counterpulsation or ventricular-assist } \\
\text { device before arrival in the anaesthetic room; acute renal failure (anuria or oliguria }<10 \mathrm{ml} / \mathrm{h} \text { ) }\end{array}$ & $3(0.2)$ \\
\hline \multicolumn{3}{|l|}{ Cardiac-related } \\
\hline CCS angina class $=4$ & CCS class 4 angina (inability to perform any activity without angina or angina at rest) & $183(9.3)$ \\
\hline Preoperative atrial fibrillation or flutter & Within 2 weeks & $8(0.4)$ \\
\hline NYHA class III or IV & NYHA classification & $1189(60.2)$ \\
\hline LVEF & Assessed by echocardiography (measured before surgery) & \\
\hline$\leq 35 \%$ & - & $226(11.4)$ \\
\hline $35 \%<E F<45 \%$ & - & $940(47.6)$ \\
\hline$\geq 45 \%$ & - & $810(41.0)$ \\
\hline \multicolumn{3}{|l|}{ Operation-related } \\
\hline Other than isolated CABG & Operations 'heavier' than the isolated CABG & $432(21.9)$ \\
\hline Aortic surgery & Combined with aortic surgery & $34(1.7)$ \\
\hline Non-elective surgery & Not routine admission for operation & $102(5.2)$ \\
\hline On-pump surgery & With extracorporeal circulation & $1214(61.4)$ \\
\hline
\end{tabular}

HF) and measured by echocardiogram, and who underwent CABG at Fuwai Hospital from January 2012 to December 2017. Detailed information on patient demographics, preoperative risk factors, operative risk factors, and in-hospital mortality was collected. The data collected included 24 preoperative factors and four operative parameters (Table 1).

All data were carefully examined. Patients under 18 years of age and those with incomplete data records were excluded. Among the patients, two were excluded because of incomplete data. Both had no ejection fraction values. In total, 1976 patients were analyzed. These patients were randomly divided into two subsets with a 3:1 ratio, according to the method used to develop the EuroSCORE II model [Nashef 2012]. These subsets were the developmental data set $(\mathrm{N}=1479)$, which was used to construct the risk model, and the validation subset $(\mathrm{N}=497)$, which was used to test and validate the model. SPSS 20.0 (SPSS Inc., Chicago, Illinois, USA) was used to perform the statistical analysis.

In the developmental data set, all the possible risk factors associated with early mortality were screened (univariable analysis) using chi-square tests for categorical covariates and t-tests or Wilcoxon rank sum tests for continuous covariates. Variables with $P$ values $>0.1$ were excluded. A stepwise multivariable logistic regression was performed using the backward 
Table 2. Risk factors for multivariable analysis

\begin{tabular}{|c|c|c|}
\hline Variable & Definition & $P$-value \\
\hline \multicolumn{3}{|l|}{ Patient-related } \\
\hline Female & - & .046 \\
\hline BMI & Body mass index & .001 \\
\hline Increased serum creatinine & $\begin{array}{l}\text { Measured before surgery or any pre- } \\
\text { vious serum creatinine>176 } \mu \mathrm{mol} / \mathrm{L}\end{array}$ & .004 \\
\hline Previous cardiac surgery & $\begin{array}{l}\text { One or more previous major cardiac } \\
\text { operation involving opening the } \\
\text { pericardium }\end{array}$ & $<.001$ \\
\hline \multicolumn{3}{|l|}{ Cardiac-related } \\
\hline $\begin{array}{l}\text { Preoperative atrial } \\
\text { fibrillation or flutter }\end{array}$ & Within 2 weeks & $<.001$ \\
\hline NYHA class III or IV & NYHA classification & .01 \\
\hline LVEF & $\begin{array}{l}\text { Assessed by echocardiography } \\
\text { (measured before surgery) }\end{array}$ & \\
\hline$\leq 35 \%$ & - & .004 \\
\hline $35 \%<\mathrm{EF}<45 \%$ & - & .017 \\
\hline$\geq 45 \%$ & - & .013 \\
\hline \multicolumn{3}{|l|}{ Operation-related } \\
\hline Other than isolated CABG & $\begin{array}{l}\text { Operations 'heavier' than the } \\
\text { isolated CABG }\end{array}$ & $<.001$ \\
\hline Aortic surgery & Combined with aortic surgery & $<.001$ \\
\hline Non-elective surgery & Not routine admission for operation & $<.001$ \\
\hline
\end{tabular}

method (criteria for entry and removal of variables were 0.05 and 0.10 ) with one variable eliminated at a time. The stability of the model was checked every time a variable was eliminated. When all statistically non-significant variables were eliminated, the goodness of fit of the final model was tested using the Hosmer-Lemeshow statistic. The logistic equation used was:

$$
\text { Predicted mortality }=\frac{e^{\left(\beta 0+\Sigma \beta i X_{i}\right)}}{1+e^{\left(\beta 0+\Sigma \beta i X_{i}\right)}}
$$

$\beta 0$ is the constant of the logistic regression equation. $\beta \mathrm{i}$ is the coefficient of the variable Xi. PGLANCE risk factors and their coefficients are detailed in Table 3. The area under the ROC curve (AUC) was utilized to assess the discriminatory power.

The discriminatory power was then tested in the validation data set. Here, we compared predicted mortality (respectively calculated by PGLANCE, EuroSCORE, EuroSCORE II, and SinoSCORE) to the observed value to evaluate their prediction accuracy.

\section{RESULTS}

A total of 1976 patients were analyzed. Elective procedures were $94.8 \%$, and isolated CABG accounted for
Table 3. Final risk factors by multivariable regression for the model

\begin{tabular}{lcc}
\hline Variable $(\mathrm{Xi}=1)$ & Coefficient $(\beta \mathrm{i})$ & $P$-value \\
\hline Female & 0.972 & .03 \\
Increased serum creatinine & 1.933 & .027 \\
Previous cardiac surgery & 1.993 & $<.001$ \\
NYHA class III or IV & 1.388 & .003 \\
LVEF & & \\
$\leq 35 \%$ & 2.011 & .005 \\
$35 \%<E F<45 \%$ & 1.516 & .017 \\
Other than isolated CABG & 0.866 & .048 \\
Aortic surgery & 1.682 & .016 \\
Constant $(\beta 0)$ & -6.553 & $<.001$
\end{tabular}

$78.1 \%$. Overall in-hospital mortality was $1.4 \%$. The mean age was 60.1 years old, with a standard deviation of 9.1, and $85.2 \%$ were male. The mean BMI was $24.9+3.9$ with $62.0 \%>24$ and $0.76 \%<18$. A history of hypertension occurred in $53.3 \%$ of patients. Diabetes was present in $25.4 \%$ of patients.

Risk factors identification: The demographics and risk factors for univariable analysis were listed in Table 1. Univariable analysis demonstrated that a number of risk factors are associated with increased mortality. These are detailed in Table 2. Multivariable analysis identified seven risk factors related to in-hospital mortality. All the independent risk factors and their coefficients $\beta i$ are listed in Table 3.

Risk model construction: Using the above risk factors listed in Table 3, the final logistic model was constructed from the developmental data set. The calibration of the model was satisfactory as the $P$ value of the HL test was $0.311(P>.05)$. The model was then applied to the validation data set with very satisfactory results (Table 4).

Risk model application: When applied to the validation data set, the area under ROC curve (AUC) of PGLANCE was 0.847 (95\% confidence interval $0.723-0.971)$, indicating very good discrimination. The discrimination powers of different models characterized by AUC are listed in Table 4. It suggests that PGLANCE had a better discrimination than EuroSCORE and SinoSCORE, similar to EuroSCORE II (0.847 versus 0.878$)$. Figure 1 displays the ROC curves of this model validated in the validation data set (Figure 1). Figure 2 displays the ROC curves of this model validated in the whole cohort (Figure 2). This system, which is similar to the EuroSCORE II, is logistic.

In the validation data set and the entire population (developmental and validation), predicted mortalities of different models are listed in Table 5. When compared with observed mortality, we could confirm that PGLANCE predicted more accurately than the other three models whose predicted mortalities were significantly higher than the observed value. 


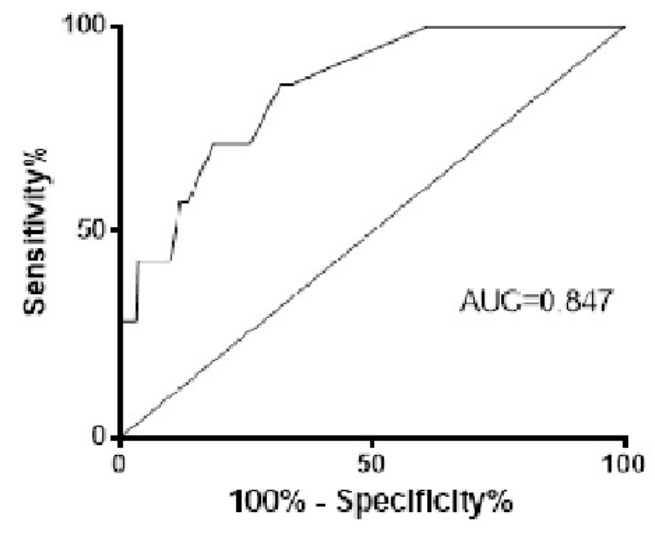

Figure 1. [MISSING]

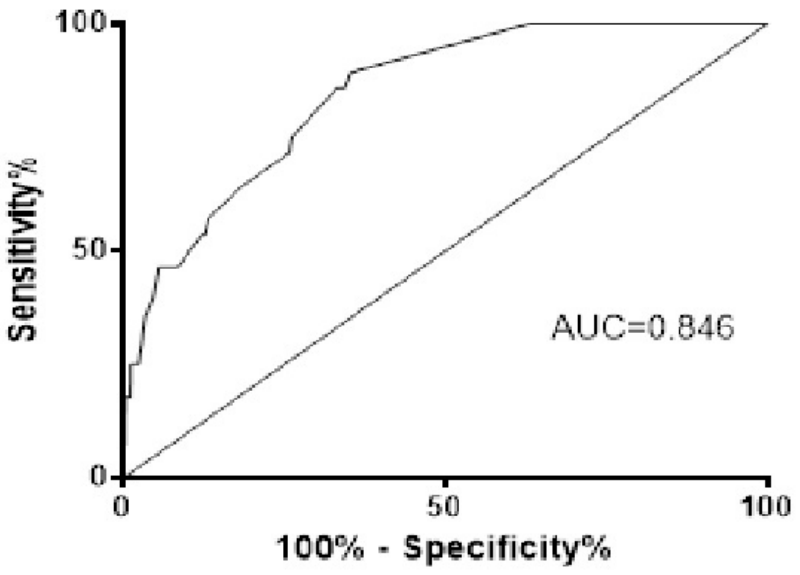

Figure 2. [MISSING]

\section{DISCUSSION}

Over the past decade, an increasing number of impaired EF or heart failure patients have undergone CABG. Chinese CABG Registry [Zheng 2012] and a survey from India [Varma 2014] have reported that about $10 \%$ of all the CABG patients have EF values less than 50\%. However, few predictive models have been specially applied to certain patients. Some of the famous models remarkably have overpredicted mortality, according to our study (Table 5). This may severely affect any critical assessment of clinical results and may lead to an incorrect therapeutic decision, necessitating the development of a more appropriate model.

EuroSCORE was one of the most successful predictive models of cardiac surgery 10 years ago, but its ability to stratify the risk has been criticized. It has been proven to markedly over-estimate individual patient risk [Choong 2009; Zheng 2009]. Correspondingly, in 2011, EuroSCORE investigators developed a revised version, EuroSCORE II, which soon was confirmed to improve the quality of clinical research and patient care. The updating from EuroSCORE to EuroSCORE II implies that logistic modeling is the best technique for mortality prediction in cardiac surgery. In this study, according to the method adopted in EuroSCORE II construction, we set up a logistic model that identified the risk factors for in-hospital mortality of impaired EF (LVEF < $50 \%$ ) patients undergoing CABG surgery.

The strength of our model and database was that our series was most recent, consecutive, large and that patients came from the whole country, which could represent Chinese characteristics of CAD. In addition, our study pertinently collected information on the patients whose EF was less than $50 \%$. Consequently, it was supposed to outperform other models for mortality prediction among certain patients.

Focusing on impaired EF patients, we used the most recent data that could exactly reflect the levels of current techniques and skills to set up the model. PGLANCE incorporated seven variables, which all turned out to be related to in-hospital mortality and included in previous models [Nashef 2012; Zheng 2013]. Unlike EuroSCORE II, which has 21 variables for mortality calculation, PGLANCE with just seven variables is easier to use and worthy of clinical popularizing. It is our opinion that better categorization of the EF value might contribute to an improved predictive ability among impaired EF patients. In terms of EF categorization, EuroSCORE II divided EF values ( $<50 \%)$ into three categories: moderate $(31-50 \%)$, poor $(21-30 \%)$ and very poor $(20 \%$ or less). In European countries, it obtained a good fit as a result of clinical use. Unfortunately, in China, patients with $\mathrm{EF}<30 \%$ seldom undergo surgery. In our study, only $67(3 \%)$ of CABG patients had an EF value of $30 \%$ or less, let alone those whose EF values were $20 \%$ or less $(\mathrm{N}=3)$. Considering the notable difference between subgroups' sample sizes if we adopted EuroSCORE II's categorization, the EF values of newly divided patients using PGLANCE were below 50\% into three categories: $<35 \%, 36-45 \%$, and $>45 \%$, which can be attributed to experience with some previous research [Nagendran 2013; Mark 2011] and balance between sample sizes of different groups. Besides, significant differences in mortality were revealed between groups $(P<.01)$, indicating that in CABG patients with impaired EFs, a lower EF value implies a poorer prognosis. Subclassification of the EF values tailored for Chinese CABG patients contributes to a better predictive capacity of the risk model.

To evaluate the predictive capability of the new model, we compared PGLANCE to EuroSCORE, EuroSCORE II, and SinoSCORE in the validation data set. PGLANCE performed better in predicting the in-hospital mortality of certain patients (Table 4 and Table 5). The AUC value of PGLANCE in the validation cohort was 0.847, which is higher than EuroSCORE and SinoSCORE, and similar to EuroSCORE II. But in terms of prediction accuracy, PGLANCE had significant benefit over the other three models.

EuroSCORE II, as an improved edition of the original EuroSCORE, collected prospective risk and outcome data on more than 20,000 consecutive patients undergoing major 
Table 4. Discrimination powers of different models

\begin{tabular}{lcccc}
\hline & PGLANCE & EuroSCORE & EuroSCORE II & SinoSCORE \\
\hline Validation set (AUC) & 0.847 & 0.775 & 0.878 & 0.698
\end{tabular}

Table 5. Mortalities predicted by different models

\begin{tabular}{|c|c|c|c|c|c|}
\hline Total & 1.42 & $1.26-1.57$ & $4.07-4.28$ & $2.30-2.54$ & 7.61-7.99 \\
\hline
\end{tabular}

cardiac surgery in 154 hospitals in 43 countries. However, in our study, the predicted mortality $(95 \% \mathrm{CI})$ for the validation data set by EuroSCORE II was 2.09-2.61\% (whole cohort $2.30-2.54 \%$ ), which is significantly higher than the observed mortality $(1.41 \%$ with validation and $1.42 \%$ with whole cohort), indicating that EuroSCORE II cannot accurately predict mortalities of Chinese impaired EF patients undergoing CABG. This result may be attributed to two reasons. First, like many other models, EuroSCORE II was based on western populations. Unlike European institutions, in our center, CABG patients with risk factors like non-elective surgery [5.2\% versus $23.3 \%$ (compared with EuroSCORE II database)], critical preoperative state $(0.2 \%$ versus $4.1 \%)$, and female gender ( $14.8 \%$ versus $30.9 \%)$ were less. Moreover, the patients in our database were younger, with a mean age of 60.1 years whereas the average age was 64.7 years in EuroSCORE II. The characteristics of Chinese CABG patients imply a lower mortality. Similar results were drawn by a previous study [Zheng 2009]. The difference between populations highlights the need to construct a model for Chinese patients. Second, nearly all of the existing risk models, such as the model of the Society of Thoracic Surgeons (STS), EuroSCORE II and SinoSCORE, were based on general CAD populations, not specific for impaired EF patients.

SinoSCORE, as the only published model based on a Chinese population, also markedly over-predicted mortality (Table 5) and had poorer AUC values. This result may be because SinoSCORE does not categorize EF values which were less than $50 \%$ as well as its old data. Theoretically, it cannot discriminate patients with impaired EF well, let alone provide accurate prediction.

Our study also had some limitations: (1) This is a singlecenter study. Further multi-center studies with larger sample sizes are needed. The model may be improved by refining and modifying some of the risk factors and the way the model handles them. In spite of the single-center research, pending further studies, we thought this model would fit in the Chinese population for two reasons. First, our patients came from across the whole country, making the data representative of the entire Chinese population. Second, given the narrowing gaps of the operative and perioperative managing techniques between large cardiac centers in China, as the largest cardic center, our institution is likely to represent the national level. (2) The results of this study were not validated using external data, thus, the PGLANCE model should be applied to non-Chinese populations with great caution. (3) We utilized in-hospital mortality as the major endpoint rather than 30-day mortality, which was used in EuroSCORE [Nashef 1999]. However, the use of in-hospital death as the endpoint is justified by the following reasons. First, other studies also used in-hospital mortality [Hannan 2006; Carosella 2009]. Second, the length of postoperative hospital stay is much longer in China than the US and Europe: the mean postoperative length of stay (PLOS) in our study population was 13.5 days, whereas a study that utilized data (496 797 procedures from 1997 to 2001) from the STS database had a mean PLOS of 6.9 days [Peterson 2002].

\section{REFERENCES}

Carosella VC, Navia JL, Alruzzeh S, et al. 2009. The first Latin-American risk stratification system for cardiac surgery: can be used as a graphic pocket-card score. Interact Cardiovasc Thorac Surg. 9(2):203-208.

Choong CK, Sergeant P, Nashef SA, Smith JA, Bridgewater B. 2009. The EuroSCORE risk stratification system in the current era: how accurate is it and what should be done if it is inaccurate? Eur J Cardiothorac Surg. Jan 35(1):59-61.

Edwards FH, Grover FL, Shroyer AL, Schwartz M, Bero J. 1997. The Society of Thoracic Surgeons National Cardiac Surgery Database: current risk assessment. Annals of Thoracic Surgery. 63(3):903-8.

F WSKPA. 2014. ESC/EACTS Guidelines on myocardial revascularization: The Task Force on Myocardial Revascularization of the European Society of Cardiology (ESC) and the European Association for CardioThoracic Surgery (EACTS) Developed with the special contribution of the European Association of Percutaneous Cardiovascular Interventions (EAPCI). Eur Heart J. 35(37):78.

Gardner SC, Grunwald GK, Rumsfeld JS, et al. 2004. Comparison of short-term mortality risk factors for valve replacement versus coronary artery bypass graft surgery. Annals of Thoracic Surgery. 77(2):549-56.

Hannan EL, Wu C, Bennett EV, et al. 2006. Risk stratification of inhospital mortality for coronary artery bypass graft surgery. Journal of the 
American College of Cardiology. 47(3):661-668.

Hu S, Zheng Z, Wu Q. 2000. Risk factors of in-hospital mortality of CABG patients: a Chinese experience. 28.

Mark DB, Knight JD, Velazquez EJ, et al. 2011. Quality-of-Life Outcomes in Surgical Treatment of Ischemic Heart Failure Quality-of-Life Outcomes With Coronary Artery Bypass Graft Surgery in Ischemic Left Ventricular Dysfunction: A Randomized Trial. Annals of Internal Medicine. 161(6):392-399.

Nagendran J, Norris CM, Graham MM, et al. 2013. Coronary revascularization for patients with severe left ventricular dysfunction. Annals of Thoracic Surgery. 96(6):2038-2044.

Nashef SA, Roques F, Michel P, Gauducheau E, Lemeshow S, Salamon R. 1999. European system for cardiac operative risk evaluation (EuroSCORE). Eur J Cardiothorac Surg. 16(1):9-13.

Nashef SA, Roques F, Sharples LD, et al. 2012. EuroSCORE II. Eur J Cardiothorac Surg. 41(4):734-744.

Peterson ED, Coombs LP, Ferguson TB, et al. 2002. Hospital variability in length of stay after coronary artery bypass surgery: results from the Society of Thoracic Surgeon's National Cardiac Database. Annals of Thoracic Surgery. 74(2):464-473.

Ponikowski P, Voors AA, Anker SD, et al. 2016. 2016 ESC Guidelines for the Diagnosis and Treatment of Acute and Chronic Heart Failure. European Heart Journal. 18(8):2129.
Varma PK, Kundan S, Ananthanarayanan C, et al. 2014. Demographic profile, clinical characteristics and outcomes of patients undergoing coronary artery bypass grafting - retrospective analysis of 4,024 patients. journal article. Indian Journal of Thoracic and Cardiovascular Surgery. December 01 30(4):272-277.

Velazquez EJ, Lee KL, Deja MA, et al. 2011. Coronary-Artery Bypass Surgery in Patients with Left Ventricular Dysfunction. New England Journal of Medicine. 364(17):1607-16.

Yancy CW, Jessup M, Bozkurt B, et al. 2013. 2013 ACCF/AHA Guideline for the Management of Heart Failure A Report of the American College of Cardiology Foundation/American Heart Association Task Force on Practice Guidelines. Journal of the American College of Cardiology. 128(16):240-319.

Zheng Z, Li Y, Zhang S, Hu S. 2009. The Chinese coronary artery bypass grafting registry study: how well does the EuroSCORE predict operative risk for Chinese population? Eur J Cardiothorac Surg. Jan 35(1):54-8.

Zheng Z, Zhang L, Hu S, Li X, Yuan X, Gao H. 2012. Risk factors and in-hospital mortality in Chinese patients undergoing coronary artery bypass grafting: analysis of a large multi-institutional Chinese database. J Thorac Cardiovasc Surg. 144(2):355-359.

Zheng Z, Zhang L, Li X, Hu S. 2013. SinoSCORE: A Logistically Derived Additive Prediction Model for Post-Coronary Artery Bypass Grafting In-Hospital Mortality in a Chinese Population. Frontiers of Medicine. 7(4):477-485. 